\title{
Deep mapping the real estate market in Milan (Italy)
}

\author{
Simone Gadenz ${ }^{b}$ *, Jacopo Mugnaini ${ }^{a}$, Alberto Cruccas ${ }^{a}$, Michele Bocci a, Matteo Villa ${ }^{a}$ \\ a Realitycs Jacopo Mugnaini, jacopo@realitycs.it,, Alberto Cruccas, alberto@realitycs,it, Michele Bocci, michele@realitycs.it, \\ MatteoVilla,matteo.villa@realitycs.it \\ ${ }^{b}$ Immobiliare Insights Simone Gadenz, insights@immobiliare.it \\ * Corresponding author
}

Keywords: Real Estate, Geostatistics, GeoAI, Urban Map, 3D Web Map

\begin{abstract}
:
Recent advancements in 3D web mapping brought the tridimensional web map environment to the masses. The main map service providers offer 3D map APIs to integrate a tridimensional exploratory spatial data environment in third party mash-up application. Using few lines of code, with low coding skills required, spatial data can be published on top of vector rendered or satellite 3D maps, fostering a deep context awareness. In some cases, the seamless integration with street view products allows a realistic first-person-view exploration of the local environment.
\end{abstract}

The representation of overlapping thematic layers on top of the 3D base maps shows room for improvement and many challenges are still to be faced, such as the effective representation of $2 \mathrm{D}$ polygon and linear objects, the positioning and visibility of $3 \mathrm{D}$ points, the information related to the inner of the $3 \mathrm{D}$ objects.

Property portals maintain huge repositories of real estate relevant data. During the last decade, Immobiliare.it, the leader Italian property portal, collected a deep spatial knowledge of the Italian market that supports the understanding of the property market dynamics. The three main market perspectives are:

- $\quad$ the supply, as the dynamic in space and time of the listings;

- $\quad$ the demand, in term of user searches, user preferences and leads generated;

- $\quad$ the market dynamism, as the sold properties with their details and spatio-temporal distribution.

A data link with the Cadastre ID is also maintained to exploit the information services made available by the Land Registry Offices and the Tax Authority.

In addition to this, a set of contextual geographies and KPIs describes the social and physical local environment hence supports the identification and the understanding of the main drivers of the observed market phenomena.

The Milan Real Estate Market Explorer project focused on the residential market of Milan, that during the last years has been the most active and dynamic market of the whole Italy, showing a trend of continuous growth since 2016. The aim of the project was to develop a cloud geographic application to enable the exploration of the available knowledge in a tridimensional environment fostering the multiscale and multi-environment seamless exploration of the social, urban and market information.

During the early stages of the project, an accurate data search and harmonization process laid the information foundation that was consolidated into a multitemporal spatial data-lake. A set of models, using according to the case geostatistics, econometrics, statistics and machine learning approaches, was developed to extract the knowledge from the data and to distill a new set of added value KPIs.

The design of the user experience was focused on the effective mapping of the different perspectives of the real estate market, that requires a seamless exploration at different map scales and between the outdoor and indoor environments. In order to make the geographic relations explicit, all the gathered information and derived KPIs were accurately coregistered within a unique spatial framework and represented on top of an interactive map that present the right level of generalization for each scale level. For example, considering the listing median unit price (€/sqm), it will be represented as mean neighborhood value as a polygon layer at city scale, as a block median value at neighborhood scale as a point layer of all available listing values at greater zoom levels. The seamless navigation between the outdoor environment and the indoor of the building was managed through an overlapping data frame that opens when the house number pin on the map or in the streetview environment is clicked.

During the next year the application model used for Milan will be reused to create similar applications for the main Italian cities. 